\title{
Portable Scanner of a 3D Surface of a Curvilinear Object
}

\author{
Konstantin KARAVAEV, Maxim TRUPHANOV \\ Elasizer, Kirov, Russia \\ DOI: $10.15221 / 17.102 \quad$ http://dx.doi.org/10.15221/17.102
}

\begin{abstract}
The research covers the operation principles and structure-functionorganization of a specialized optical-electronic device for calculation of a volumetric object.

Here we present the results of our development of a special-purpose portable scanner of a 3D surface of a complete curvilinear object with a complex surface. Favorably compared with the analogs, our scanner features a high-precisioncalculation of a curvilinear object along with a compact and simple technical design which makes it easy to manufacture. We providedifferent technical solutions of our scanner based on novel original decisions which shuffle its technical features depending on the objectiveand enable to apply it in various ways. The above-said makes our scanner commercially attractive for certain tasks required in the market of customized items for individual application.

The general advantages of the designed scanner are several: the use of several passive optical-electronic sensors which are placed in preset fixed positions and factory calibrated with no additional settings necessary; use of commercially available electronic components; low time-consuming acquisition of parameters of a 3D surface; and ease of operation.

The experiments we conducted showed that, depending on the interrelated position of the device and the analyzed object, our device gives the calculation error of the 3D coordinates of about $\pm 1.5 \mathrm{to} \pm 2.5 \mathrm{~mm}$.
\end{abstract}

Keywords: 3D body scanning, multisensor vision, machine vision, distortion.

\section{Introduction}

The main objective in manufacturing personalized bodywear, footwear, protective wear for extreme sports and dangerous occupations is to provide high-precision calculation of the surface and anatomic features of a person's head and body. While meeting this objective the special attention is paid to the task of constructing of the easy-to-use and commercially profitable scanner, which can be implemented for customized protective outfit and provides an adequate accuracy. Experiments show that the accuracy sufficient for practical application ranges from 2 to $5 \mathrm{~mm}$.

The proposed scanner is intended primarily to measure the shape of the head, yet, with minimal changes in design, it can be used to measure the shape of other parts of the body, such as an ankle or a foot.

The operating principle of our scanner consists of the following basics: assured tight fitting of the contact elastic surface with contrast-colored markers to the measured object; calculation of geometric parameters of the surface through analyzing the positions of the markers.

The multisensory scanner we introduce consists of two main components:

- mechanical system which guarantees the tight fitting of the elastic contact surface to the object (e.g. elastic contact cap on the head) by creating the vacuum in the enclosed space around the object to provide best contact of the elastic surface with the measured object due to the impact of the atmospheric pressure with the following acquisition of the image of the surface with multiple sensors in order to calculate the coordinates of the markers in space; and

- electronic computing system to read and measure the parameters of the markers.

The mechanical system consists of the airtight box in which a part of the measured object is inserted, the electric pump and the elastic contact surface. The electronic computing system consists of several optical-electronic sensors, sensor switching system, and the computation block.

We developed several solutions for the computation system of the scanner and the layout of the optical-electronic sensors:

- variant \#1 uses 4 sensors and the system of 6 mirrors which provide acquisition of the image of the object from all sides for the following calculation of the 3D shape of the object;

- variant\#2 uses 12 to 36 sensors (depends on the size and complexity of the shape of the measured object) connected pairwise in a quick scan mode which provide calculation of 3D coordinates of marker points on the elastic contact surface in order to calculate the parameters of the object;

Our multisensor optical-electronic scanner is designed to be used for analyzing the shape of human body and body parts in various anthropometric researches. 


\section{Functional features of the designed system}

The scanner includes a system of video cameras located in predesigned positions on a metal frame. To make the measurements, the frame with cameras is placed over the head (or leg) of a customer (figs. 1, 2).

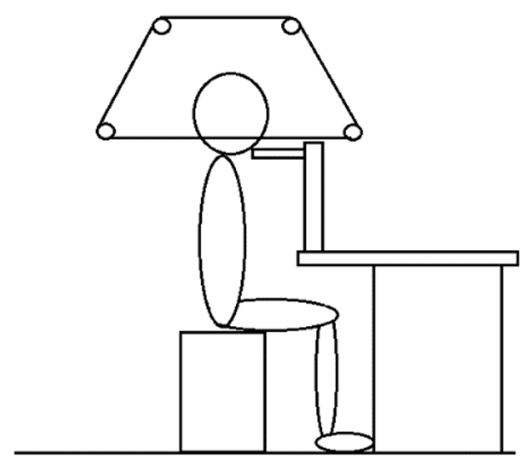

Fig. 1. Schematic side view of the scanner

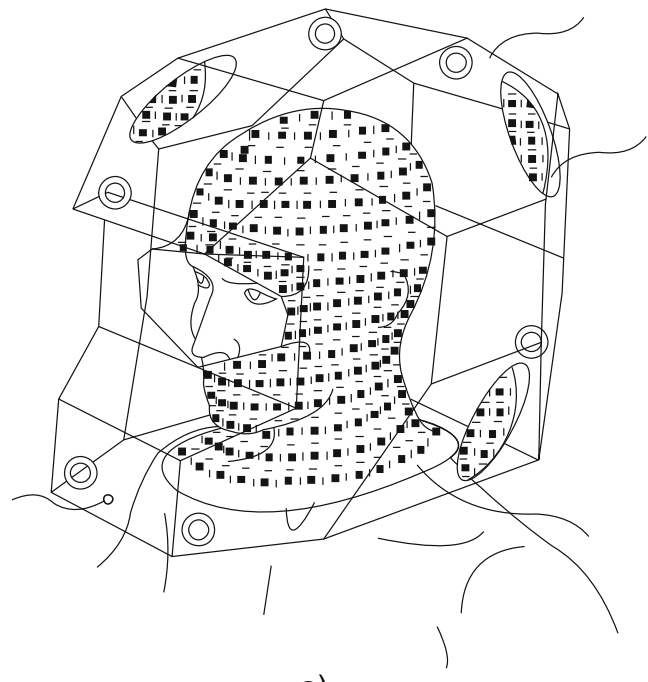

a)

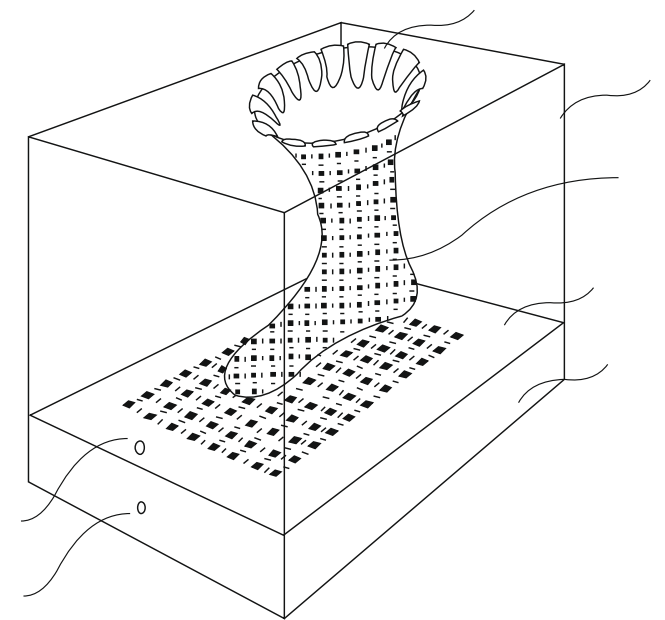

b)

Fig. 2. Two variants of measurable objects: a) a head (for customized helmet), b) a leg (for customized shoes)

The measurement principle consists of the following operations:

- acquirethe 2Dcoordinates from each pair of adjacent cameras,

- calculate 3D coordinates of each point on the surface of the head

- combine the acquired 3D data from each pair of cameras into a continuous system of points which approximate the surface of the head,

- iteratively specify 3D coordinates of the points by bringing the acquired system to the form that complies the closed shape approximating the head,

- transfer the results of measurements to the 3D printer of a special format file to produce the elements of a customized protective gear (i.e. motorcycle helmet) or a headwear (i.e. lady's hat).

The measurement procedure takes about 30 seconds and the major part of this time is taken by the explanation to the client about the proper positioning of the head during the procedure. The measurement itself takes from 500 to 1200 milliseconds depending on the anatomic features of the head and on how still the client is during the procedure.

Prior the procedure the one-time factory calibration of the scanner is performed which consists in the calibration of the relative positioning of the optical-electronic sensors.

For calibration, a tested object is positioned in the visual field of all cameras and the parameters of the positions of optical-electric sensors are calculated automatically and saved as a configuration file for further use during operation of the scanner.

For a computing device, a laptop or a PC is used. 
The scanner operates in two task-oriented modes:

- the first mode for high-precision measurement of the surface of an object,

- the second mode for faster measurement of the surface of an object with slightly less accuracy in comparison to the first mode.

The first mode is based on creation of discharged air pressure around the measured object which provides a very tight fit of a special marker surface perfectly outlining the shape of the measured object. The optical-electronic device reads the passive optical markers without contact and according to the above-mentioned approach calculates the precise $3 \mathrm{D}$ coordinates of the points on the surface followed by the construction of the continuous surface.

The second mode does not use the discharged air around the object which significantly simplifies the design and makes it less costly. The fitting of the contact object is realized by means of its elastic properties.

The first mode has been tested and is recommended for calculation of the parameters of feet and ankles for production of customized footwear. The second mode is preferable for production of protective headgear and headwear.

\subsection{Operation principle of the designed system}

Fig. 3 illustrates the layout of videocameras.

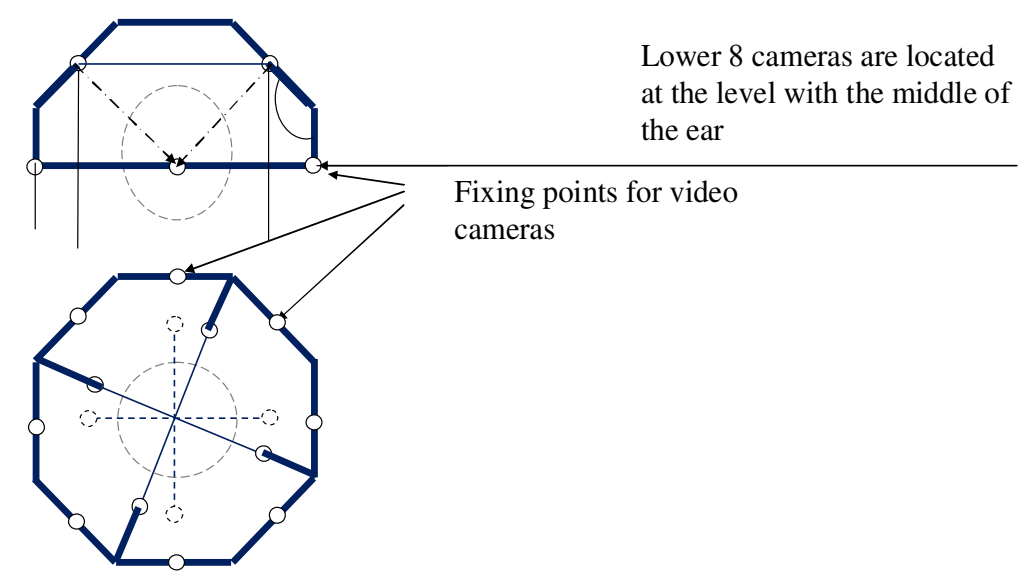

Fig. 3. Videocameras positions

The calculation principle of 3D coordinates is covered in our earlier publication [1].

Now we present the principles of calibration which precision highly influences the operation of the scanner.

Unlike traditional approaches, the calibration method we have developed allows to use an easy-to-manufacture object to obtain a precise conformity of local systems of optical-electronic sensors and create a unified coordinate system from multiple optical-electronic sensors [2].

Once the interconnection of local coordinate systems is established the scanner is ready to operate. In the operation mode the way to measure the configuration and size of an object that has volume is as follows:

- measure the distance between the optical-electronic sensors in the measurement system and the surface of the elastic contact coating which has markings; to do so the measured surface of an object is placed in the space (for the first variant of scanner use which involves creation of discharged air around an object) approximated to the surface of the elastic coating of the device with markings; after measurement the obtained data is processed and the virtual model of the measured surface is created.

The designed device consists of several interconnected modules including the system for measuring the configuration and dimensions of an object that has volume, the elastic coating with passive markings on the inner surface, the compressor to create the required pressure inside the closed module. To create a fit contact between the measured surface of an object and the above-mentioned device, the pressure is created necessary for the coating with markings to be pressed to this surface.

Next, by means of the above-mentioned system of optical-electronic sensors the configuration and the dimensions of the object are calculated, the information on the location of the markers on the inner side of the coating is read and processed followed by creation of the digital model with repeats the configuration, dimensions, and shape. 


\section{Experimental results}

Currently we are in the process of conducting experimental research and finalizing the design of the scanner with consideration of the modifications which need to be done.

Resent experiment show that the measurement accuracy of coordinates when the vacuum system is used ranges from 0.7 to $1.5 \mathrm{~mm}$. When the contact marker surface is used, the accuracy ranges from 1.5 to $2.5 \mathrm{~mm}$ which is also adequate for practical application for manufacturing customized protective gear, bodywear and footwear.

\section{References}

[1] Karavaev, K. Multisensor optical-electronic device for calculation of surface parameters of the 3D curvelinear object / K. Karavaev, M.Truphanov // Proceedings of the 7th International Conference on 3D Body Scanning Technologies, Lugano, Switzerland, 30 Nov.-1 Dec. 2016, P. 279-285.

[2] Titov, V The calibration method for stereoscopic vision system [text] / V. Titov, S. Degtiarev, M. Truphanov // Machine graphics and vision. Poland, Vol. 17, No. 4, 2008. - pp. $373-387$.

[3] Viksten, F. Point-of-InterestDetectionforRangeData [Text] / Viksten, F. Nordberg, K. Kalms, M. // Pattern Recognition, 2008. ICPR 2008. 19th International Conference on Publication Date: 8-11 Dec. 2008, P.1-4 